\title{
Reversible Photodimerization of Ester Derivatives of Thymine Having Long Alkyl Chains in Solid Film
}

\author{
Eiko MochIZukI, ${ }^{*}$ Norimitsu TohnaI, Yuhua WANG, Takao SAITo, Yoshiaki Inaki, ${ }^{\dagger}$ \\ Mikiji MrYaTA, Nobuyoshi YASUI, ${ }^{*}$ and Yasushi KaI* \\ Department of Material \& Life Science, Osaka University, \\ Yamadaoka 2-1, Suita, Osaka 565-0871, Japan \\ * Department of Materials Chemistry, Osaka University, \\ Yamadaoka 2-1, Suita, Osaka 565-0871, Japan
}

(Received November 4, 1999)

\begin{abstract}
Reversible photodimerizations of ester derivatives of thymine having long alkyl chains were studied in thin solid film. The spin coated thin film of thymine compounds gave the photodimer under irradiation of UV light at 280 $\mathrm{nm}$, and photodimers gave original thymine compounds by UV irradiation at $240 \mathrm{~nm}$. The rates of photodimerization in thin solid film increased with length of the alkyl chain, followed by decrease when the carbon number of the alkyl chain was above 13. The behavior of photodimerization depended on the carbon number of the alkyl chains. The structures of the thymine derivatives in thin solid film were estimated from the data of differential thermal analysis, powder X-ray diffraction, and crystal structure of the single crystal. The layer structures of thymines and alkyl chains were concluded to be the reason for photoactivity in thin solid film. When the carbon numbers of the alkyl chains were 12 and 13 , the photodimerizations were fast in the thin solid film, but diminished by annealing of the film. The reason for this was the formation of the inactive micro crystals by annealing.
\end{abstract}

KEY WORDS Thymine / Photodimerization / Photosplitting / Differential Scanning Calorimetry / Powder X-Ray Diffraction / Crystal Structure / Solid Film /

DNA is damaged by irradiation of UV light. Photoactive nucleic acid bases are pyrimidine bases such as thymine, cytosine and uracil, but purine bases are stable toward UV irradiation. ${ }^{1}$ The photodimerization of thymine is reversible depending on wavelength of irradiating UV light, although photodimerization of uracil is irreversible (Figure 1). ${ }^{2}$ Polymer and monomer derivatives of thymine may thus be used as reversible photosensitive materials such as reversible photoresists and reversible photo-recording systems. ${ }^{3-8}$

The intermolecular photodimerization of thymine bases grafted onto the side chain of polymethacrylate led to photocrosslinking of the polymers, thus allowing them to be applied as negative type photoresists. ${ }^{9}$ The orientation of thymine bases was found important for intermolecular photodimerization in preference to the intramolecular one. Bis-thymine compounds where two thymines were connected by ester or amide bonds were also negative type photoresists, because the photodimerization of thymine bases gave the polymers in solid film. ${ }^{10}$ For photodimerization of the bis-thymine compounds in solution, however, the main photoproduct was the cyclic photodimer. The conformation of the bis-thymine com-<smiles>Cc1c[nH]c(=O)[nH]c1=O</smiles>

Thymine
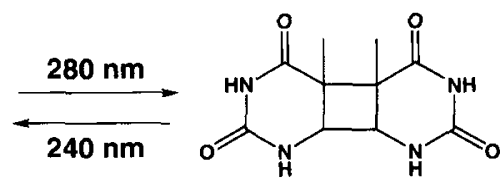

Photodimer
Figure 1. Reversible photodimerization of thymine bases. pounds was concluded to be an important factor for photodimerization. Polyamides and polyesters containing thymine photodimers in the main chain were negative type photoresists. ${ }^{11-12}$ Irradiation of UV light at short wavelength $(240 \mathrm{~nm})$ on these polymers gave low molecular weight compounds containing thymine bases soluble in alkaline aqueous solution.

For high sensitive and completely reversible photoactive materials, it is necessary to study reversible photodimerization and conformations of the thymine derivatives in solid film. There are troublesome problems for these studies. For investigation of photodimerization, thymine compounds are necessary to give transparent film of amorphous state. Single crystals are necessary for studies of conformation by X-ray structure analysis. Ester derivatives of thymine having long alkyl chain in this paper (Figure 2) are special cases for the study of structure and reversible photodimerizations in solid film. Single crystals of the ester derivatives were obtained for the X-ray structure analysis, and transparent solid films were obtained for photodimerization. This paper deals with the photodimerization of the ester derivatives of thymine in solid film, and structure of thin solid film studied by differential scanning calorimetry (DSC) and powder X-ray diffraction.

To find the relationship between photodimerization of thymine derivatives and structures in solid state, the photodimerization of thymine base in single crystal was studied for 1-alkylthymine derivatives. ${ }^{13-17}$ Four crystal forms were obtained for 1-alkylthymines from various solvents. The crystals from ethyl acetate ${ }^{14}$ and acetonitrile $^{16}$ gave photodimers of thymine bases, but crystals from ethanol ${ }^{15}$ and $N, N$-dimethylformamide ${ }^{17}$ did not

\footnotetext{
${ }^{\dagger}$ To whom correspondence should be addressed (E-mail: inaki@chem.eng.osaka-u.ac.jp).
} 


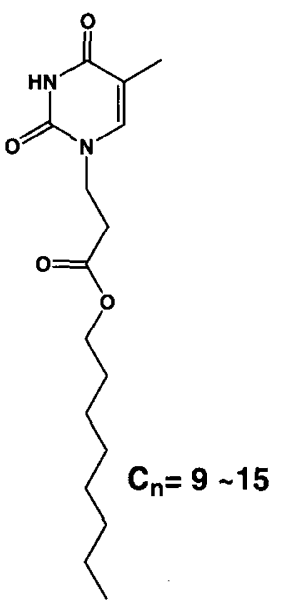

Figure 2. Ester derivatives of thymine.

give the photodimer. From the structures of photodimers obtained from the single crystal, thymine bases in crystal were concluded to give the photodimer by disrotatory motion in the crystal. The mobility of the thymine bases in crystal was concluded necessary for the photodimerization of the thymine bases in crystals. The single crystals were obtained for ester derivatives of thymine having long alkyl chains, but the crystals did not give the photodimer. The terminal methyl group of the alkyl chain was concluded to inhibit the rotation of thymine bases in crystal during the photodimerization process. ${ }^{18}$ The crystal structures of the ester derivatives, however, may give information on structures of thin solid films.

\section{EXPERIMENTALS}

\section{Materials}

The ester derivatives of thymines, alkyl 2-(thymin-1yl)propionates, were prepared from 2-(thymin-1-yl)propionic acid and long alkyl alcohols using 1,3dicyclohexylcarbodiimide. ${ }^{18}$

\section{Photoreactions}

Thin solid film was obtained by spin coating on a quartz plate from chloroform solution $\left(0.74 \mathrm{~mol} \mathrm{~L}^{-1}\right)$, followed by drying overnight under reduced pressure. Polymer film was obtained by spin coating on a quartz plate from chloroform solution ( $10 \mathrm{wt} \%$ ) of poly(vinyl acetate) (molecular weight was 140000) with the thymine compounds, followed by drying overnight under reduced pressure. Molar ratio of the thymine compound to poly. (vinyl acetate) was $1: 3$.

Irradiation of UV light was carried out by a spectro irradiator (Nihon Bunko, CRM-FA) at $280 \mathrm{~nm}$ for photodimerization and at $240 \mathrm{~nm}$ for splitting reactions. The energy irradiated was $8.81\left(\mathrm{~mJ} \mathrm{~cm}^{-2}\right)$ at $280 \mathrm{~nm}$ and $1.35\left(\mathrm{~mJ} \mathrm{~cm}^{-2}\right)$ at $240 \mathrm{~nm}$ per one count. Photodimerization was followed by measuring UV spectra at $270 \mathrm{~nm}$ (Nihon Bunko, UV/VIS 600).

\section{Instruments}

DSC was measured by a Seiko I\&E DSC6200 series. $\mathrm{X}$-ray powder diffraction patterns were measured by a Rigaku X-ray diffractometer RINT 2000 with $\mathrm{Cu}-K_{\alpha}$ ra-

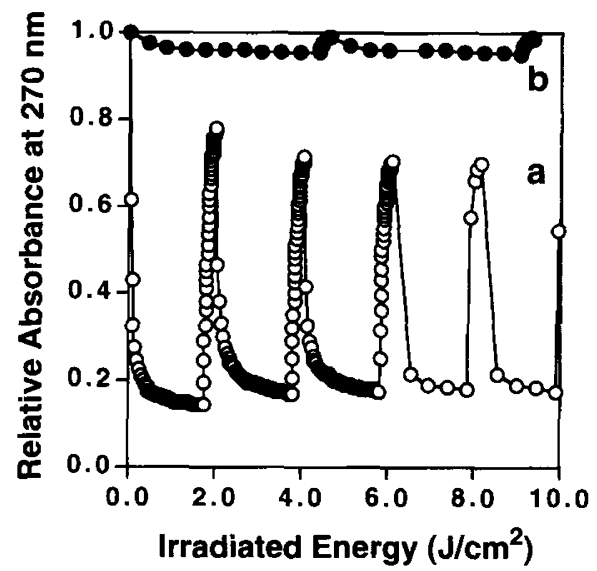

Figure 3. Typical repeated photodimerization of tridecyl 2(thymin-1-yl)-propionate (C13) in thin solid film. (a) Spin coat film, and (b) after annealing at $100^{\circ} \mathrm{C}$.

diation at room temperature.

\section{RESULTS}

\section{Photodimerization in Thin Solid Film}

Reversible photodimerization of ester derivatives of thymines was studied in thin solid film prepared by spin coating from chloroform solution. Figure 3 shows typical repeated photodimerization of tridecyl (C13) ester derivatives of thymine in thin solid film. The absorbance of UV spectra at $270 \mathrm{~nm}$ decreased by irradiation at 280 $\mathrm{nm}$, indicating the formation of the photodimer and increased again by irradiation at $240 \mathrm{~nm}$, suggesting photosplitting of the photodimer (a in Figure 3). After annealing the thin solid film at $100^{\circ} \mathrm{C}$, photodimerization was found to hardly occur, suggesting structural change of the thin solid film (b in Figure 3).

In the case of photodimerization by monochromatic light at $280 \mathrm{~nm}$, the thymine monomers (M) gave the photodimer (D) quantitatively without by-product (eq 1). The energy of the irradiated light to thymine (E) is proportional to reaction time, thus leading to eq 2 , where $A_{0}$ and $A_{t}$ are UV absorption at $270 \mathrm{~nm}$, at time $=0$ and $t$, respectively. Plots by eq 2 for the data in Figure 3 gave Figure 4.

$$
\begin{gathered}
2 M \frac{k}{-\frac{\mathrm{d}[\mathrm{M}]}{\mathrm{d} t}=k[\mathrm{M}]} \\
\ln \frac{A_{t}}{A_{0}}=-k^{\prime} E
\end{gathered}
$$

Figure 4 shows the photodimerization of the decyl ester $(\mathbf{C 1 0})$ in the thin solid film $(\mathbf{a}-\mathbf{c})$ with the data in the polymer film (d) and in solution (e). The rate of photodimerization in chloroform solution was slow but the process proceeded completely as shown in Figure 4(e) for decyl 2-(thymin-1-yl)-propionate (C10). Photodimerization of thymine derivatives in solution proceeds by way of triplet state. ${ }^{2}$ Short lifetime of the singlet state and inefficient intersystem crossing are the reasons of slow reaction for the photodimerization of thymine in solution. 


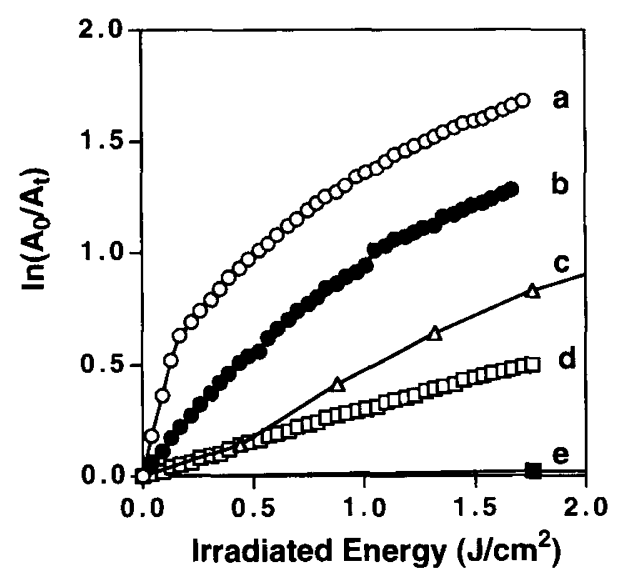

Figure 4. Conversion of photodimerization for decyl 2-(thymin-1yl)-propionate (C10) in various states under UV irradiation at 280 nm. (a) Spin coat film, (b) after photo splitting, (c) after annealing at $100^{\circ} \mathrm{C}$, (d) in poly(vinyl acetate) film, and (e) in chloroform solution.

The photodimerization was studied in the polymer film using poly(vinyl acetate) (PVAC). A polymer film was obtained from chloroform solution of the ester derivatives of thymine and poly(vinyl acetate) by spin coating on a quartz plate. The rate of photodimerization in poly(vinyl acetate) (d in Figure 4) was faster than the rate in chloroform solution. In the polymer matrix, photodimerization occurs from associated thymine bases via singlet state with higher quantum yield than that of triplet state.

Photodimerization in the thin solid film was faster than in solution and polymer film, suggesting suitable orientation of the thymine bases in the thin solid film. In the case of the spin coated film (a in Figure 4), the rate was very fast at the beginning, and slowed down later. Thymines in suitable orientation for photodimerization gave the photodimer rapidly and thymines in unsuitable orientation for photodimerization gave no photodimer. The second photodimerization after photosplitting of the photodimer slowed down as shown in $\mathbf{b}$ of Figure 4. Thymines in suitable orientation for photodimerization became unsuitable by moving molecules during photodimerization. Similar transformation of the structure was observed for thin solid film after heat treatment (c in Figure 4).

Repeated reversible photodimerization in the spin coat film was studied for nonyl (C9), decyl (C10), undecyl (C11), dodecyl (C12), tridecyl (C13), tetradecyl (C14), and pentadecyl (C15) 2-(thymin-1-yl)-propionates. Decay of photodimerization was observed except $\mathbf{C 1 2}$ and C13. The conversion of first photodimerization from $\mathbf{C 9}$ to $\mathbf{C 1 5}$ is plotted against irradiated energy in Figure 5. Up to about $0.5 \mathrm{~J} \mathrm{~cm}^{-2}$ of irradiated energy, the photodimerization was fast, and the curve rounded to a slow rate. The initial fast reaction rates of $[\mathbf{C 1 0}, \mathbf{C 1 1}]$ were similar to [C9], but the slow reactions above $0.5 \mathrm{~J} \mathrm{~cm}^{-2}$ of $[\mathbf{C 1 0}, \mathbf{C 1 1}]$ were faster than [C9]. The initial rates of the first photodimerization of $[\mathbf{C 1 2}, \mathbf{C 1 3}]$ were very fast, and conversion of $\mathbf{C 1 3}$ reached $90 \%$ within $0.5 \mathrm{~J} \mathrm{~cm}^{-2}$. For $[\mathbf{C 1 4}, \mathbf{C 1 5}]$, the initial rates were faster than for [C10, C11], but final conversion was lower than for $[\mathbf{C 1 0}, \mathbf{C 1 1}]$. Then the rates of the first photodimerization

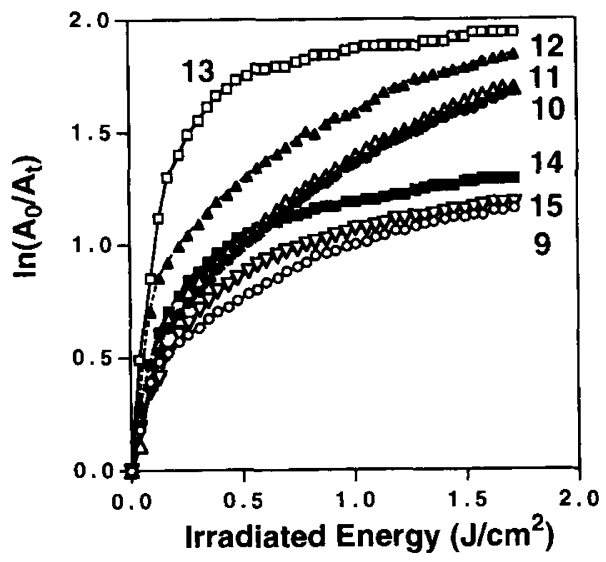

Figure 5. Conversion of first photodimerization in the spin coat film from C9 to C15. UV irradiation: $280 \mathrm{~nm}$. The numbers are carbon numbers of alkyl chain.

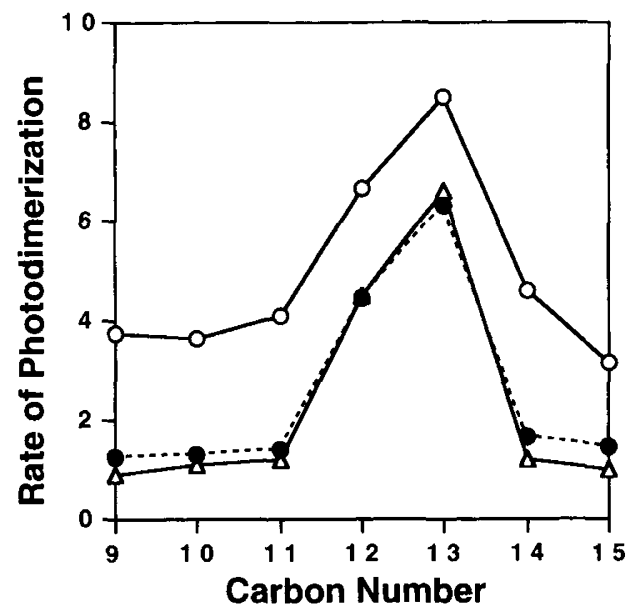

Figure 6. Initial rate of photodimerization against carbon number of alkyl chains. ( $\bigcirc)$ First, $(O)$ second, and $(\triangle)$ third photodimerizations. Rate: $\mathrm{cm}^{2} \mathrm{~J}^{-1}$.

in Figure 5 may represented as [C9], [C10, C11], [C12, C13], and [C14, C15] according to the bending mode of the curve.

The initial rates of the first photodimerization in Figure 5 were calculated using eq 2 , and plotted against carbon number of the alkyl chains in Figure 6. The figure shows interesting results of the highest reactivity for C13. The rates of the second and third photodimerizations after photosplitting were calculated and plotted in Figure 6. The rates of the second and third photodimerizations were slower than the rates of the first photodimerization. However, the dependency of the rate of photodimerization on the carbon number of the alkyl chains was similar for the first, second, and third photodimerizations. The equation for the calculation of rate was based on the assumption that all thymine bases in the film form the photodimer. However, Figure $3 \mathrm{a}$ of C13 indicates that about $20 \%$ of thymine bases could not form the photodimer in thin solid film. When the regenerated thymine bases by photosplitting reaction were used as initial concentrations of thymine bases in eq 2 , the rates of the second and the third photodimerizations were similar to the rate of the first photodimerization. 


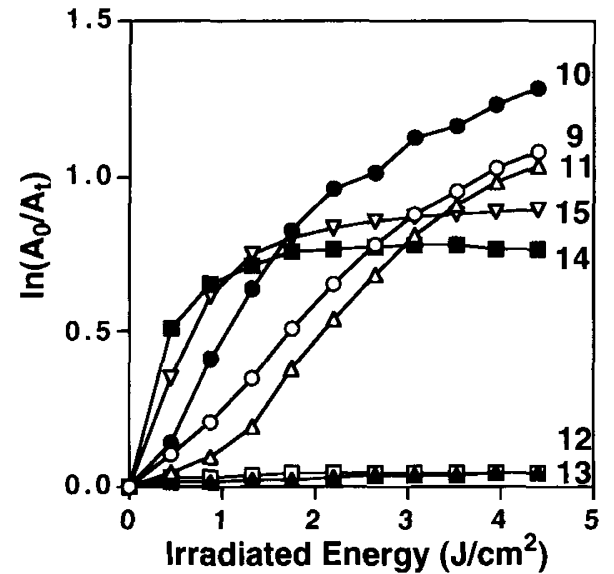

Figure 7. First photodimerization after annealing at $100^{\circ} \mathrm{C} . \mathrm{UV}$ irradiation: $280 \mathrm{~nm}$. Numbers are carbon numbers of alkyl chains.

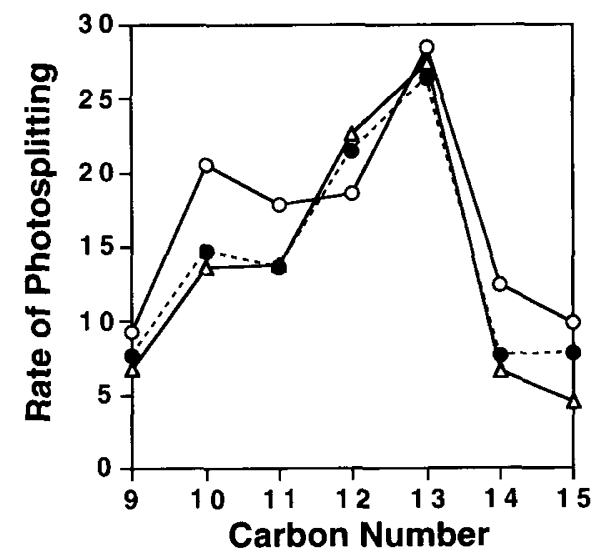

Figure 8. Initial rate of photosplitting against carbon number of alkyl chains. $(\bigcirc)$ First, $(\bigcirc)$ second, and $(\triangle)$ third photosplitting UV irradiation: $240 \mathrm{~nm}$. Rate: $\mathrm{cm}^{2} \mathrm{~J}^{-1}$.

The unreacted thymines in the film should be in the inactive crystal state formed during photodimerization by reorientation of molecules in the thin solid film.

\section{Photodimerization in the Annealed Solid Film}

The spin-coated thin films were annealed at $100^{\circ} \mathrm{C}$ above melting points. The first photodimerization after annealing is shown in Figure 7. The reactivities of $\mathbf{C 1 0}$ and $\mathbf{C 1 1}$ decreased by annealing to become the same as C9. The curves in this figure are thus classified as $[\mathbf{C 9}$, C10, C11], [C12, C13], and [C14, C15]. A remarkable change observed for $\mathbf{C 1 2}$ and $\mathbf{C 1 3}$ after annealing was the disappearance of photodimerization, suggesting that the structure of the thin film is transformed by annealing.

\section{Photosplitting of Photodimer in Thin Solid Film}

The thymine photodimers formed by UV irradiation at $280 \mathrm{~nm}$ in the thin solid film were split into original thymines by UV irradiation at $240 \mathrm{~nm}$. Figure 8 shows the results of photosplitting from $\mathbf{C 9}$ to $\mathbf{C 1 5}$. The fastest reaction of photosplitting was observed for C13. The dependence of the first photosplitting on carbon number in Figure 8 was similar to that of the photodimerizations in Figure 6, but the rates for $\mathbf{C 1 0}$ and $\mathbf{C 1 1}$ were fast com-

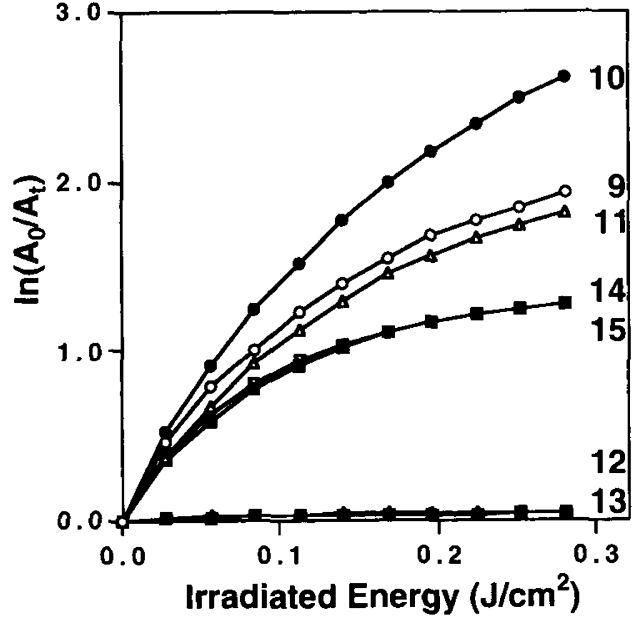

Figure 9. Conversion of photosplitting of the photodimer in annealed film from C9 to C15. UV irradiation: $240 \mathrm{~nm}$. Numbers are carbon numbers of alkyl chains.

pared with the photodimerization.

Photosplitting of Photodimer in Annealed Solid Film

Photosplitting of the photodimer formed in the annealed film gave irregularity for $\mathbf{C 1 2}$ and $\mathrm{C13}$ (Figure 9). Figure 9 shows smooth increase of conversion and slow reactions of $\mathbf{C 1 2}$ and $\mathbf{C 1 3}$. It is uncertain whether small decrease of the absorbance for the annealed thin film of $\mathrm{C12}$ and $\mathrm{C13}$ by UV irradiation at $280 \mathrm{~nm}$ corresponds to the formation of the photodimer. The data of $\mathrm{C12}$ and $\mathrm{C13}$ in Figure 9 are thus not reliable.

The results of photodimerization and photosplitting in thin solid film bring up three questions: type of photodimerization reaction, fastest reaction for $\mathbf{C 1 3}$, and vanishing of the photodimerization by annealing for $\mathbf{C 1 2}$ and C13. These questions are answered by crystal structure, DSC and X-ray powder diffraction for thin solid film as follows.

\section{Structurse of Ester Derivatives of Thymine in Thin Solid Film}

The results of photodimerization for the ester derivatives of thymine suggest the presence of several structures in thin solid film. Differential scanning calorimetry (DSC) measurements were carried out for crystals obtained from benzene solution. Measurement was carried out for single crystals, for solid after slow cooling $\left(3^{\circ} \mathrm{min}^{-1}\right)$ of the melt, and for solid after quick cooling $\left(100^{\circ} \mathrm{min}^{-1}\right)$ of the melt.

Figure 10 shows DSC curves for C9. The melting point of crystals from benzene solution was $84.6^{\circ} \mathrm{C}$, but the melting point after slow cooling was $64.0^{\circ} \mathrm{C}$ and melting points after quick cooling were 67.4 and $75.6^{\circ} \mathrm{C}$. The crystal structure of single crystals from benzene may thus be transformed into another unstable structure by melting. Figure 11 shows X-ray powder diffraction patterns for C9. The thin film cast from chloroform solution (a) was not amorphous but had a layer structure where the distance between thymine layers was $21.12 \AA$ and the distance between alkyl chains was $4.20 \AA$. Pattern (a) was different from that of the single crystal from benzene (c). The sample after annealing at $100^{\circ} \mathrm{C}$ (melting) 


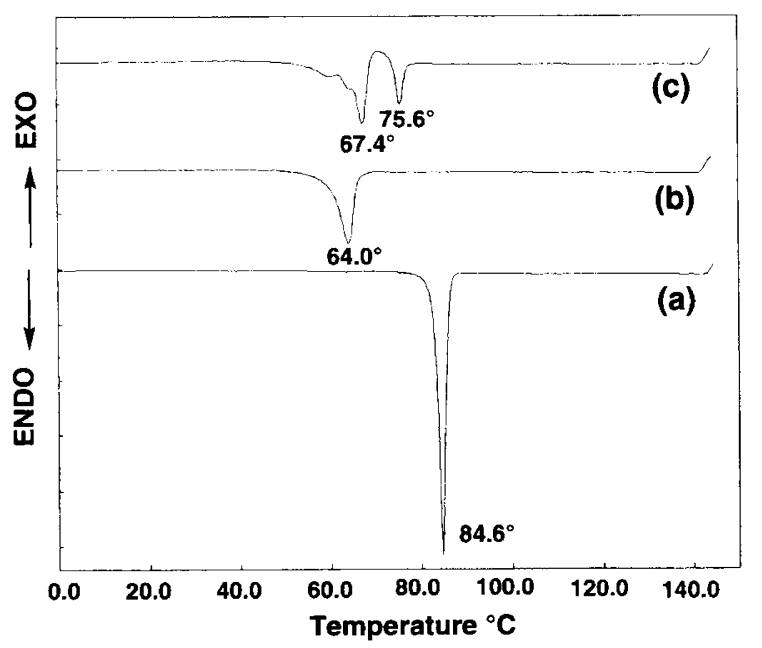

Figure 10. DSC curves for C9. Heating: $5^{\circ} \mathrm{min}^{-1}$. (a) Single crystals, (b) after slow cooling $\left(3^{\circ} \mathrm{min}^{-1}\right)$ of the melt, (c) after quick cooling $\left(100^{\circ} \mathrm{min}^{-1}\right)$ of the melt.

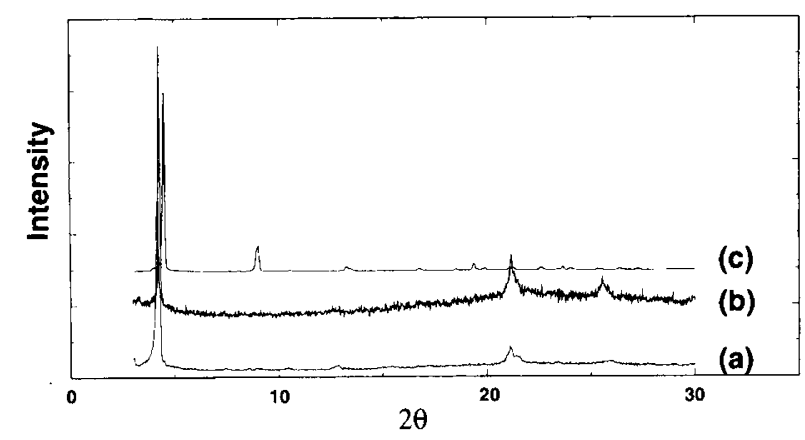

Figure 11. X-ray powder diffraction patterns for C9. (a) Thin film cast from chloroform solution, (b) after melting of single crystals, and (c) single crystals from benzene.

(b) showed a similar pattern as cast film (a). It was thus concluded that the structure of cast film was not the structure of the single crystal but the structure of unstable crystal melted at $64.0^{\circ} \mathrm{C}$.

The patterns of DSC curves for C10 and C11 were similar as shown in Figure 12 for $\mathbf{C 1 0}$. The peak at 89.4 ${ }^{\circ} \mathrm{C}$ of the single crystal (a) split into three peaks at $63.6^{\circ}$, $76.3^{\circ}$, and $88.5^{\circ} \mathrm{C}$ by slow cooling of the melt (b). Quick cooling of the sample (c) gave also three peaks $\left(67.2^{\circ}\right.$, $76.7^{\circ}$, and $87.1^{\circ} \mathrm{C}$ ), but the ratios of these peaks were different from that at slow cooling. The results suggested that the single crystal became a mixture of crystals by melting. The melting point of C11 single crystal was $90.8^{\circ} \mathrm{C}$ and the peak split into three peaks at $79.0^{\circ}, 86.1^{\circ}$, and $90.0^{\circ} \mathrm{C}$ by slow cooling of the melt. In the X-ray powder diffraction patterns (Figure 13 for $\mathbf{C 1 0}$ ), the cast film from chloroform solution (a) showed small peaks of crystal and strong peaks of interplaner spacing at $21.3 \AA$ and $4.18 \AA$. However, small peaks of crystal were different from the peaks of single crystals (c from benzene, and $\mathbf{d}$ from ethanol). Melting of the solid film (b) gave no peak of crystals but strong peaks of interplaner spacing at 21.3 $\AA$ and $4.18 \AA$ suggesting the formation of a layer structure. The X-ray powder diffraction patterns for $\mathbf{C 1 1}$ was similar to the pattern of C10, except the values of spacing. It was thus concluded that the structures of the

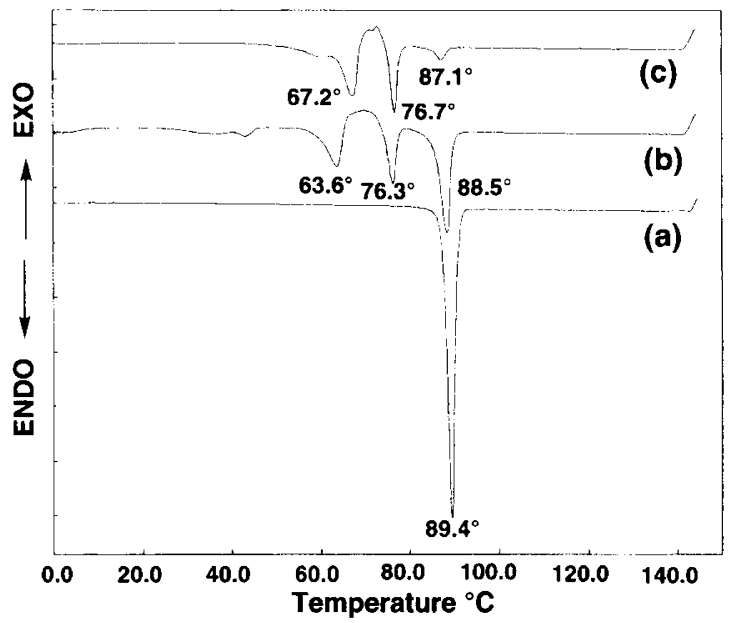

Figure 12. DSC curves for C10. Heating: $5^{\circ} \mathrm{min}^{-1}$. (a) Single crystals, (b) after slow cooling $\left(3^{\circ} \mathrm{min}^{-1}\right)$ of the melt, and (c) after quick cooling $\left(100^{\circ} \mathrm{min}^{-1}\right)$ of the melt.

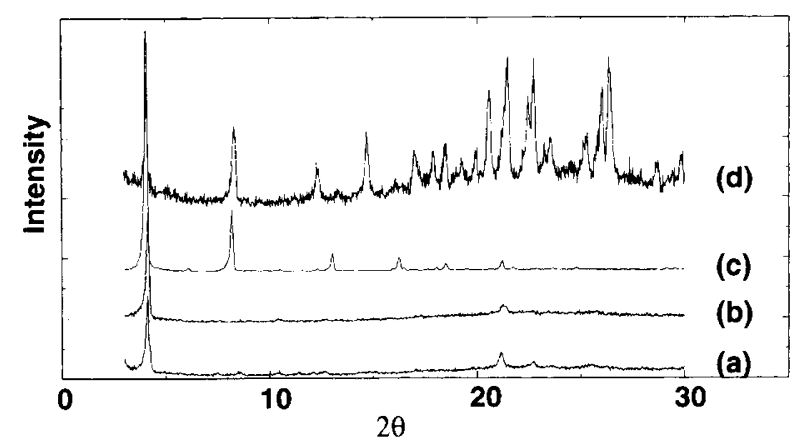

Figure 13. X-ray powder diffraction patterns for C10. (a) Thin film cast from chloroform solution, (b) after annealing at $100^{\circ} \mathrm{C}$ (melting), (c) single crystals from benzene, and (d) single crystals from ethanol.

cast film for the group $[\mathbf{C 1 0}, \mathbf{C 1 1}]$ were not those of single crystals but layer structures.

DSC curves for $[\mathbf{C 1 2}, \mathbf{C 1 3}]$ are similar as shown in Figure 14 for C12. The melting points of the single crystal for $\mathrm{C12}$ and $\mathrm{C13}$ were $95.3^{\circ} \mathrm{C}$ and $95.8^{\circ} \mathrm{C}$, respectively. A broad exothermic peak was shown at $54.2{ }^{\circ} \mathrm{C}$ in addition to a sharp endothermic peak at $94.7^{\circ} \mathrm{C}$ for the slow cooling sample of $\mathbf{C 1 2}(\mathbf{b})$. The exothermic peak around $54^{\circ} \mathrm{C}$ should be the melting of amorphous parts to form crystals. The slow and quick cooling are thus the same hydrogen bonding structures of thymines but give amorphous parts by aggregation of alkyl chains. The compounds in this group gave similar patterns of the $\mathrm{X}$ ray powder diffraction. In X-ray powder diffraction patterns (Figure 15 for C12), small peaks of cast film from chloroform solution (a) are the same as the peaks of single crystal from benzene (c). The peak at $3.84(2 \theta)$ of Figure 15a was assigned to the crystal. The peak assigned to the layer structure is not shown in the Figure but should be smaller then 3.84. Melting of the film (b) gave no peak of crystals but gave strong peaks of interplaner spacing at $22.6 \AA$ and $24.8 \AA$ suggesting the formation of a layer structure. DSC and the X-ray powder diffraction for $[\mathbf{C 1 4}, \mathbf{C 1 5}]$ gave similar data for $[\mathbf{C 1 2}$, C13]. The melting points of the single crystals for C14, 


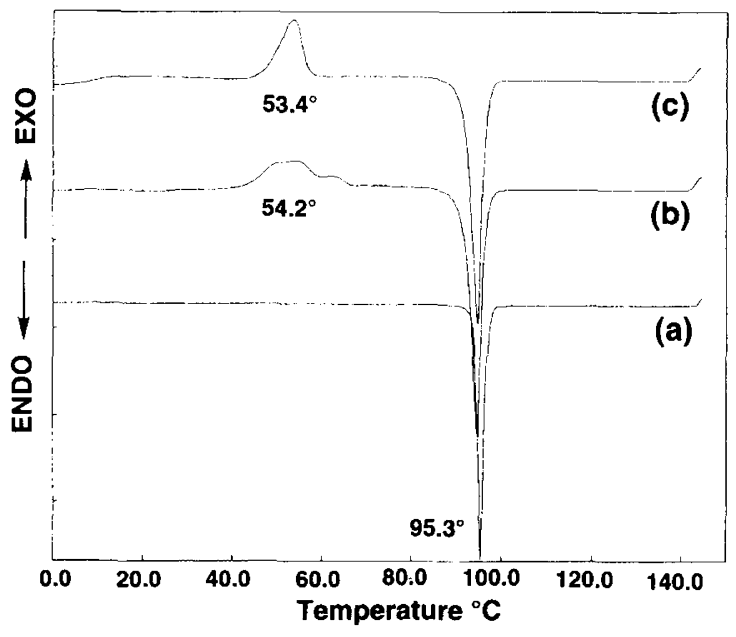

Figure 14. DSC curves for C12. Heating: $5^{\circ} \mathrm{min}^{-1}$. (a) Single crystals, (b) solid after slow cooling $\left(3^{\circ} \mathrm{min}^{-1}\right.$ ) of the melt, and (c) solid after quick cooling $\left(100^{\circ} \mathrm{min}^{-1}\right)$ of the melt.

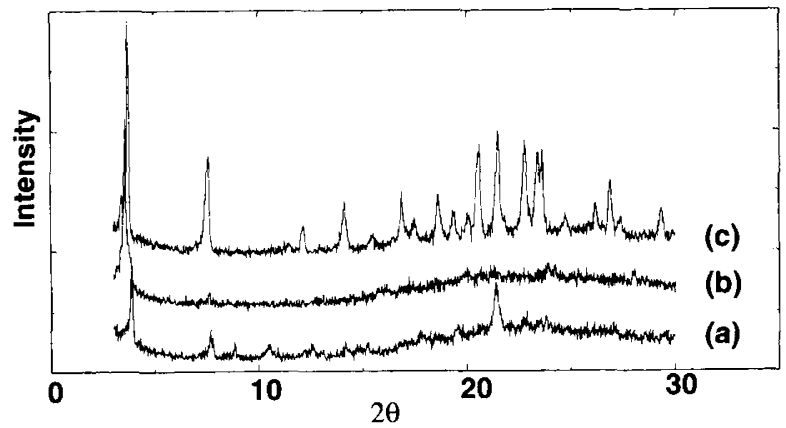

Figure 15. X-ray powder diffraction patterns for C12. (a) Thin film cast from chloroform solution, (b) after melting of the film, and (c) single crystals from benzene.

and $\mathrm{C15}$ were $97.2^{\circ} \mathrm{C}$ and $99.0^{\circ} \mathrm{C}$, respectively. The structures of the cast film for the groups $[\mathbf{C 1 2}, \mathbf{C 1 3}]$ and $[\mathbf{C 1 4}, \mathbf{C 1 5}]$ are thus a mixture of single crystals and layer structures.

\section{DISCUSSIONS}

\section{Crystal Structure and Photodimerization of Thymine}

\section{Compounds}

Single crystals of ester derivatives of thymine having long alkyl chains were obtained from benzene, chloroform and ethanol solutions. The crystal structures were analyzed by X-ray crystal analysis. Single crystal did not give the photodimer by irradiation of UV light, while the spin-coat thin film underwent photodimerization.

Figure 16a shows the crystal structure of $\mathbf{C 1 3}$ obtained from benzene solution. ${ }^{18}$ Molecular arrangement in this structure was similar to the structure of photoactive crystal of 1-alkylthymine obtained from acetonitrile (Figure 16b). ${ }^{16}$ The most important factor determining activity for photodimerization seems the distance between thymine rings. Certainly, differences in crystal structures for two crystals were in packing of the thymine bases. Figure 17 shows arrangement of the hydrogen bonded thymine bases in the crystals. Distance between planes of the hydrogen-bonded thymines was around
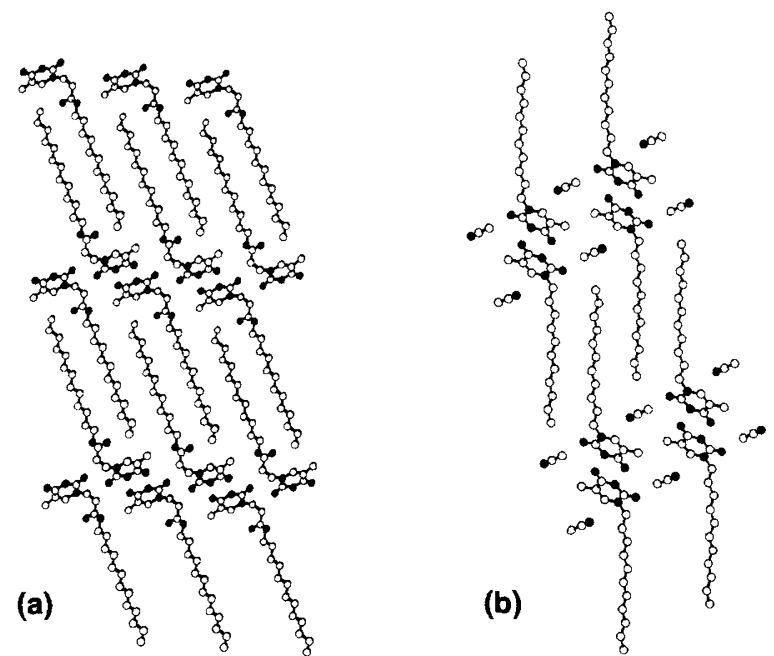

Figure 16. Crystal structure of (a) $\mathbf{C 1 3}$ obtained from benzene solution, ${ }^{18}$ and (b) photoactive crystal of 1-undecylthymine (R11) obtained from acetonitrile. ${ }^{16}$

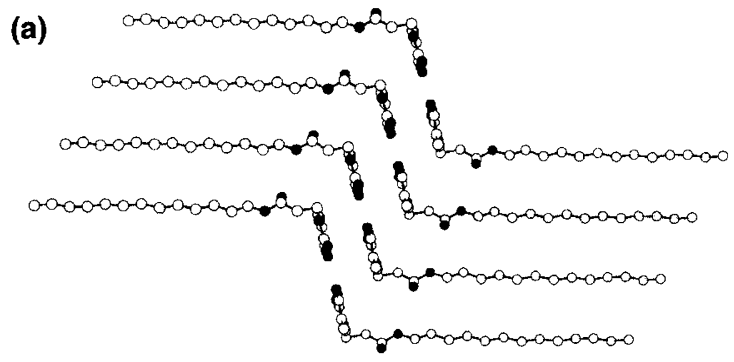

(b)

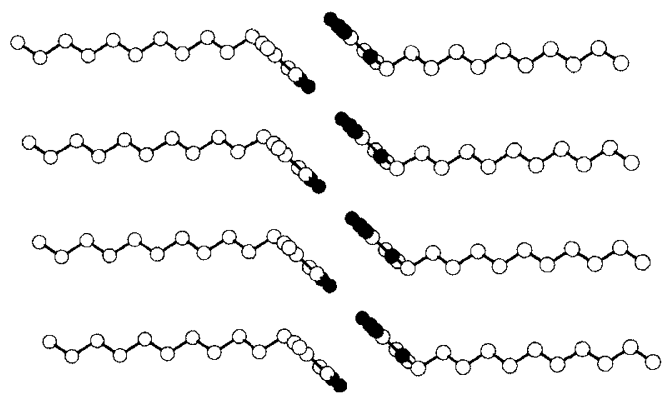

Figure 17. Arrangement of hydrogen bonded thymine bases. (a) C13 obtained from benzene solution, ${ }^{18}$ and (b) 1-undecylthymine (R11) obtained from acetonitrile. ${ }^{16}$

$3.4 \AA$ for both crystals. Thymine bases slide obliquely and hardly overlap each other for C13 (Figure 17a), because the alkyl chain is perpendicular to the thymine ring. In photoactive crystals of 1-undecylthymine (R11) obtained from acetonitrile, overlapping of the thymine bases was observed (Figure 17b), because the angle was large between alkyl chain and thymine ring.

Thymine bases in the single crystal are necessary to rotate for the formation of photodimer. ${ }^{14}$ Figure 18 shows the nearest two molecules in the crystal for $\mathbf{C 1 3}$ and R11. The thymine base in the crystal obtained from acetonitrile (R11) rotates during photodimerization, because the crystal contains acetonitrile molecules and the terminal methyl group is very far from the thymine base 


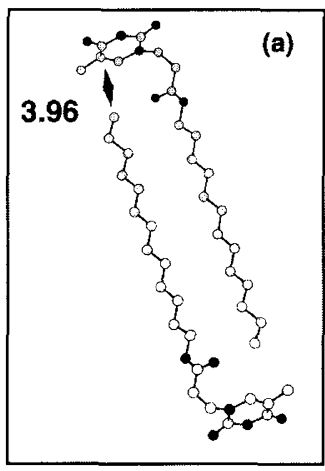

Ester (C13)

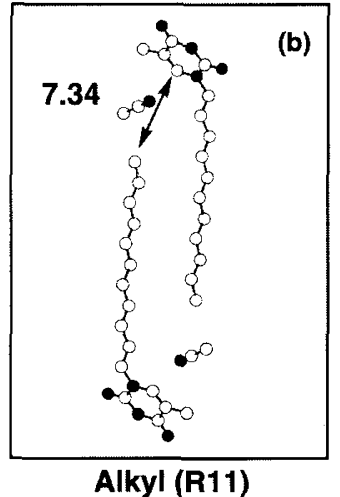

Alkyl (R11)
Figure 18. Nearest two molecules in crystals for (a) C13 obtained from benzene solution, ${ }^{18}$ and (b) photoactive crystals of 1undecylthymine (R11) obtained from acetonitrile. ${ }^{16}$

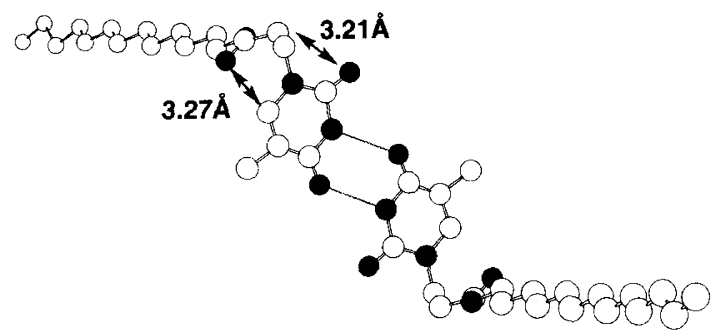

Figure 19. Hydrogen-bonded pair of the thymine compound of C13 from data of crystal structure. ${ }^{18}$

(Figure 18b). In the case of $\mathbf{C 1 3}$, however, the terminal methyl group of the alkyl chain is located close to the thymine base, where the distance was $3.96 \AA$ (Figure 18a). Therefore, the thymine base of $\mathbf{C 1 3}$ was not able to rotate for photodimerization. This is the main reason for high photoactivity of crystals of 1-alkylthymine from acetonitrile (R11) and inactivity of $\mathbf{C 1 3}$ for photodimerization.

\section{Estimation of the Structure in the Thin Solid Film}

Structures of ester derivatives of thymine in the thin solid film were determined from the data of the crystal structure, powder X-ray diffraction, and DSC. In casting from chloroform solution forming the thin film, the first interaction of the molecules should be hydrogen bonding of thymine bases. Figure 19 shows a hydrogen-bonded pair of thymine compounds for C13 from the data of crystal structure. ${ }^{18}$ The alkyl chains in $\mathbf{C 1 3}$ are perpendicular to the thymine ring because of interaction between the ester group and thymine. The pair aggregates by van der Waals interaction of the long alkyl chain to form micro crystals. Finally, the aggregation of the micro crystal forms crystals (Figure 16a) inactive for photodimerization.

DSC data are summarized in Figure 20, where [C14, C15] is the same as $[\mathbf{C 1 2}, \mathbf{C 1 3}]$. The single crystal (a) of the first [C9] melts at $84.6^{\circ} \mathrm{C}$, and the cooling forms two crystals (b) and (c) which are different from the original crystal (a). Consequently, the thin solid film of C9 contains a mixture of two crystals (b) and (c), even if crystals are formed. The data of the powder X-ray diffraction support this conclusion.

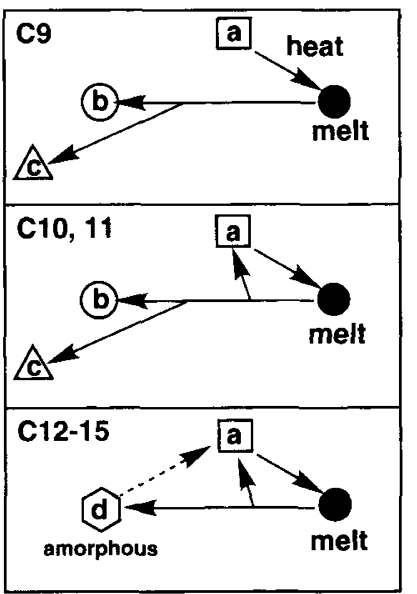

Figure 20. DSC data for ester derivatives of thymine. Original single crystals (a) melts, and forms the crystals (a,b, and c) and amorphous part (d) by cooling.

In the case of the second group [C10, C11], the melt of the single crystal (a) gives three crystals of (a), (b), and (c) by cooling. The thin solid film of this group should thus contain three crystals when crystallization occurred in the film. The data of the powder X-ray diffraction of the thin film indicated that the film was not crystals but a layer structure.

$[\mathrm{C12}, \mathrm{C13}]$ and $[\mathrm{C14}, \mathrm{C15}]$ are different from the former two. The single crystal (a) melts at around $100^{\circ} \mathrm{C}$, and forms again crystals (a) by cooling accompanying the formation of the amorphous part (d). Crystals (a) formed by cooling should be inactive for photodimerization. The data of the powder X-ray diffraction indicated that the film contained mixture of the crystals and the layer structure.

The data of powder X-ray diffraction of the thin film indicated that the film was a layer structure. The layer structure should be formed by loose interactions of alkyl chains of the hydrogen-bonded pairs. The layer structure of the film is illustrated and compared with the crystal structure for $\mathbf{C 1 3}$ (Figure 21), where the distance between layers in the thin solid film is greater than that of crystals. In the layer structure of the thin film, the rotation of the thymine base should be possible, because the distance between the thymine and the terminal methyl group of alkyl chain is long enough. The thymine compounds in the layer structure of the thin solid film are able to undergo photodimerizations. The distance of the layer structures becomes short by annealing, and photodimerization is inhibited.

\section{Photodimerizations in Thin Solid Film}

The information of the structure of the thin solid film mentioned above makes clear the reversible photodimerization of the thymine compounds having long alkyl chain in thin solid film. Photodimerization of ester derivatives of thymine in the thin film is summarized in Figure 22.

In [C9], the conversion of the second photodimerization (2nd) decreased compared with the first photodimerization (1st). The annealed film (Ann-1st) gave also the photodimer. The thin solid film of $\mathbf{C 9}$ contained unstable crystals of (b) and (c) (Figure 20). One of these 


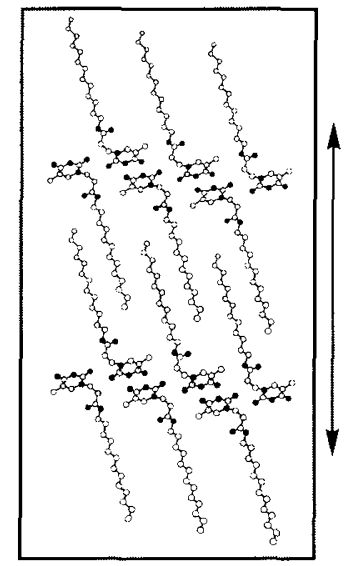

Film (illustration)

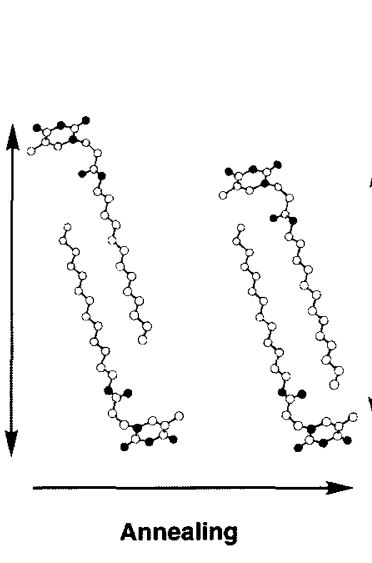

Annealing

Figure 21. Layered structure in the thin solid film and crystal structure of $\mathbf{C 1 3} .^{18}$

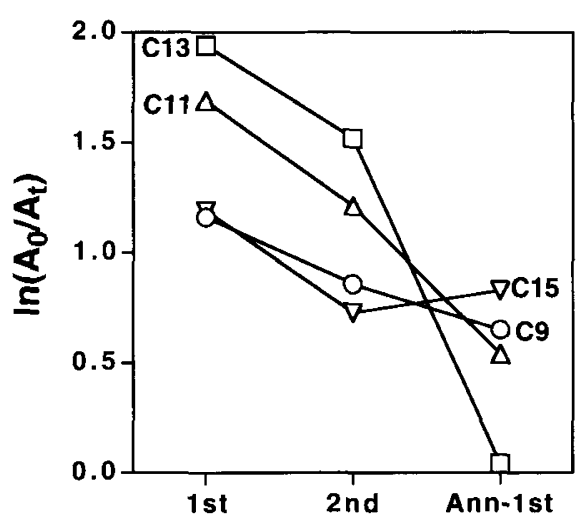

Figure 22. Outline of photodimerization of the ester derivatives of thymine in thin solid film. Conversion $\left(\ln \left[A_{0} / A_{t}\right]\right)$ plotted against first photodimerization (1st, at $1.95 \mathrm{~J} \mathrm{~cm}^{-2}$ ), second photodimerization (2nd, at $1.90 \mathrm{~J} \mathrm{~cm}^{2}$ ), and first photodimerization of annealed film (Ann-1st, at $2.20 \mathrm{~J} \mathrm{~cm}^{-2}$ ).

crystals may be active for photodimerization, and the active crystal transformed to inactive one during the first photodimerization and the annealing process.

The conversion of the first photodimerization for the second $[\mathbf{C 1 0}, \mathbf{C 1 1}]$ was higher than that of $[\mathbf{C 9}]$, because the film was the photoactive layer structure. The activity decreased in second photodimerization and decreased again after annealing of the film. In [C10, C11], inactive crystals should be formed from the photoactive layer structure during the first photodimerization and melting of thin solid film.

The thin solid films of the third [C12, C13] had high activity for the first photodimerization, but did not give the photodimer after annealing of the film. DSC and the $\mathrm{X}$-ray powder diffraction indicated the film was a mixture of crystals and layered structure. The photodimerization of thymine bases should be fast in the layered structure, where the thymine bases are arranged suitably for photodimerization and rotation of the thymines is possible. The melting of the film caused the formation of crystals inactive for photodimerization. Therefore, the photoactivity of this group disappeared after melting of the film. The formation of crystals was not found in Xray powder diffraction, because the formed crystals were too small to be detected. Some peaks such as those of single crystal, however, were found in the X-ray powder diffraction after annealing of the thin solid film below the melting point.

The compounds of the fourth $[\mathbf{C 1 4}, \mathbf{C 1 5}$ ] have longer alkyl group than the others. When the alkyl chain is long, the interaction of the alkyl chain is strong, which is supported by high melting point of the crystals. The strong interaction of the long alkyl chains may inhibit the moving of the thymine bases, which is necessary for the photodimerization. Conversion of the photodimerization thus decreases with increase of length of the alkyl chain. The first photodimerization and the melting of the thin solid film caused the formation of inactive crystals from the disordered part, but the active layer structure was maintained because of strong interaction of the long alkyl chain.

\section{Photosplitting of Photodimer in Thin Solid Film}

As shown in Figure 8, the rates of the first photosplitting increased with carbon number of the alkyl chains, and decreased after C13. Photosplitting of the thymine photodimer in solution is caused by steric repulsion of C5-methyl groups of thymine base. ${ }^{2}$ Photosplitting in solid state is caused by strain of the crystal lattice in addition to strain of the C5-methyl groups. The reason is that photodimerization of thymine bases having long alkyl chains in crystal induces rotation of thymine bases, resulting in strain in crystals as illustrated in Figure 23. When the alkyl chain become long, the strain of the crystal lattice become strong because the interaction of the alkyl chain increase with length of the alkyl chain. The rate of photosplitting reaction should increase with length of the alkyl chain. The reason for the slow reaction for $[\mathbf{C 9}]$ and $[\mathbf{C 1 4}, \mathbf{C 1 5}]$ is not clear, but low conversion of the photodimerization may not cause high strain in thin solid film. The slow photosplitting reaction of $[\mathbf{C 1 2}, \mathbf{C 1 3}]$ for annealed thin solid film (Figure 9) was uncertain, because the slight decrease of the UV absorbance by irradiation at $280 \mathrm{~nm}$ could not be identified as formation of the photodimer. 


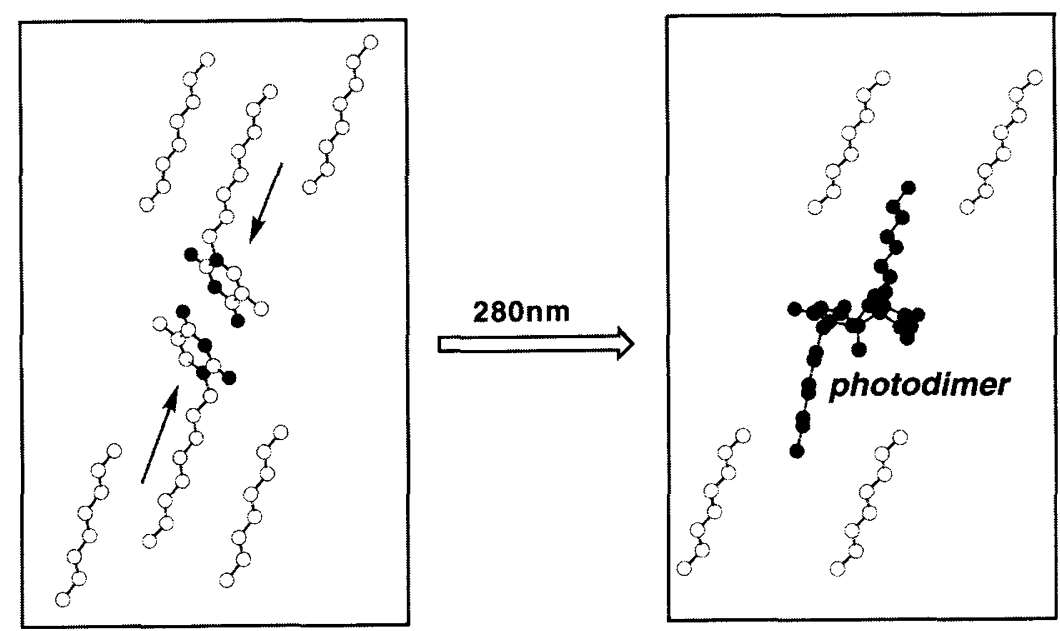

Figure 23. Induced strain during photodimerization of thymine bases in crystals (illustration).

\section{CONCLUSIONS}

Reversible photodimerizations in solid thin film were studied for ester derivatives of thymine having long alkyl chains. The rates of photodimerizations in the thin film increased with the length of the alkyl chain, but decreased above C13. The behavior of the photodimerization was represented as $[\mathrm{C9}],[\mathrm{C10}, \mathrm{C11}],[\mathrm{C12}, \mathrm{C13}]$ and $[\mathbf{C 1 4}, \mathbf{C 1 5}]$. The structure of the thin film was estimated and related to photodimerization from the data of DSC, X-ray powder diffraction, and crystal structure of the single crystal. X-ray data suggested the layer structure of thymines and alkyl chains for thin solid film, where the thymine bases rotate during photodimerization. Therefore, the ester derivatives of thymine in the thin solid film gave photodimes, but the single crystals hardly gave the photodimer.

Acknowledgments. The authors express appreciation to Profs. T. Kitayama, K. Ute and Mr. T. Kawauchi for DSC measurements.

\section{REFERENCES}

1. M. H. Patrick and R. O. Rahn, in "Photochemistry and Photobiology of Nucleic Acids," Vol. II, S. Y. Wang, Ed., Academic Press, New York, N.Y., 1976, pp 35-95.

2. G. J. Fisher and H. E. Johns, in "Photochemistry and Photobiology of Nucleic Acids," Vol. I, S. Y. Wang, Ed., Academic Press, New York, N.Y., 1976, pp 225-294.

3. Y. Inaki, M. J. Moghaddam, and K. Takemoto, in "Polymers in Microlithography Materials and Process," ACS Sym. Ser.,
412, E. Reichmanis, S. A. MacDonald, and T. Iwayanagi, Ed., American Chemical Society, Washington, D.C., 1989, pp 303 $-318$.

4. Y. Inaki, N. Matsumura, K. Kanbara, and K. Takemoto, in "Polymers for Microelectronics," Y. Tabata, I. Mita, S. Nonogaki, K. Horie, and S. Tagawa, Ed., Kodansha-VCH, Tokyo, 1990, pp 91-102.

5. Y. Inaki, N. Matsumura, and K. Takemoto, in "Polymers for Microelectronics," ACS Sym. Ser., 537, L. F. Thompson, G. Wilson, and S. Tagawa, Ed., American Chemical Society, Washington, D.C., 1994, pp 142-164.

6. Y. Inaki, Polymer News, 17, 367 (1992).

7. Y. Inaki, Y. Wang, M. Kubo, and K. Takemoto, J. Photopolym. Sci. Technol., 4, 259 (1991).

8. Y. Inaki, Y. Wang, T. Saito, and K. Takemoto, J. Photopolym. Sci. Technol., 5, 567 (1992).

9. M. J. Moghaddam, S. Hozumi, Y. Inaki, and K. Takemoto, Polym.J., 21, 203 (1989).

10. M. J. Moghaddam, K. Kanbara, S. Hozumi, Y. Inaki, and K. Takemoto, Polym. J, 22, 369 (1990).

11. M. J. Moghaddam, S. Hozumi, Y. Inaki, and K. Takemoto, $J$. Polym. Sci., Part A: Polym. Chem., 26, 3297 (1988).

12. M. J. Moghaddam, Y. Inaki, and K. Takemoto, Polym. J, 22, 468 (1990).

13. T. Sugiki, N. Tohnai, E. Mochizuki, T. Wada, and Y. Inaki, Bull. Chem. Soc. Jpn., 69, 1777 (1996).

14. N. Tohnai, Y. Inaki, M. Miyata, N. Yasui, E. Mochizuki, and Y. Kai, J. Photopolym. Sci. Technol., 11, 59 (1998).

15. N. Tohnai, Y. Inaki, M. Miyata, N. Yasui, E. Mochizuki, and Y. Kai, Bull. Chem. Soc. Jpn., 72, 851 (1999).

16. N. Tohnai, Y. Inaki, M. Miyata, N. Yasui, E. Mochizuki, and Y. Kai, Bull. Chem. Soc. Jpn., 72, 1143 (1999).

17. E. Mochizuki, N. Yasui, Y. Kai, Y. Inaki, N. Tohnai, and M. Miyata, Bull. Chem. Soc. Jpn., in press (1999).

18. N. Tohnai, Y. Inaki, M. Miyata, N. Yasui, E. Mochizuki, and Y. Kai, J.Am. Chem. Soc., in contribution. 